\title{
VERY METAL-POOR STARS IN THE OUTER GALACTIC BULGE FOUND BY THE APOGEE SURVEY
}

\author{
Ana E. García Pérez ${ }^{1}$, Katia Cunha ${ }^{2,3}$, Matthew Shetrone ${ }^{4}$, Steven R. Majewski ${ }^{1}$, Jennifer A. Johnson ${ }^{5}$, \\ Verne V. Smith ${ }^{6}$, Ricardo P. Schiavon ${ }^{7}$, Jon Holtzman ${ }^{8}$, David Nidever ${ }^{9}$, Gail Zasowski $^{5}$, Carlos Allende Prieto ${ }^{10,11}$, \\ Timothy C. Beers ${ }^{6,12}$, Dmitry Bizyaev ${ }^{13}$, Garrett Ebelke ${ }^{13}$, Daniel J. Eisenstein ${ }^{14}$, Peter M. Frinchaboy ${ }^{15}$, \\ Léo Girardi ${ }^{16,17}$, Fred R. Hearty ${ }^{1}$, Elena Malanushenko ${ }^{13}$, Viktor Malanushenko ${ }^{13}$, Szabolcs Meszaros $^{11}$, \\ Robert W. O’Connell ${ }^{1}$, Daniel Oravetz ${ }^{13}$, Kaike Pan ${ }^{13}$, Annie C. Robin ${ }^{18}$, Donald P. Schneider ${ }^{19}$, \\ Mathias Schultheis ${ }^{18}$, Michael F. Skrutskie ${ }^{1}$, Audrey Simmonsand ${ }^{13}$, and John C. Wilson ${ }^{1}$ \\ ${ }^{1}$ Department of Astronomy, University of Virginia, Charlottesville, VA 22904-4325, USA \\ ${ }^{2}$ Steward Observatory, University of Arizona, Tucson, AZ 85721, USA \\ ${ }^{3}$ Observatório Nacional, São Cristóvão, Rio de Janeiro, Brazil \\ ${ }^{4}$ McDonald Observatory, University of Texas at Austin, Fort Davis, TX 79734, USA \\ ${ }^{5}$ Department of Astronomy, The Ohio State University, Columbus, OH 43210, USA \\ ${ }^{6}$ National Optical Astronomy Observatories, Tucson, AZ 85719, USA \\ ${ }^{7}$ Gemini Observatory, 670 N. A’Ohoku Place, Hilo, HI 96720, USA \\ ${ }^{8}$ Department of Astronomy, MSC 4500, New Mexico State University, P.O. Box 30001, Las Cruces, NM 88003, USA \\ ${ }^{9}$ Department of Astronomy, University of Michigan, Ann Arbor, MI 48109, USA \\ ${ }^{10}$ Departamento de Astrofísica, Universidad de La Laguna, E-38206 La Laguna, Tenerife, Spain \\ ${ }^{11}$ Instituto de Astrofísica de Canarias, E-38205 La Laguna, Tenerife, Spain \\ 12 Department of Physics \& Astronomy and JINA, Joint Institute for Nuclear Astrophysics, Michigan State University, E. Lansing, MI 48824, USA \\ 13 Apache Point Observatory, P.O. Box 59, Sunspot, NM 88349-0059, USA \\ ${ }^{14}$ Harvard Smithsonian Center for Astrophysics, 60 Garden Street, Cambridge, MA 02138, USA \\ ${ }^{15}$ Department of Physics and Astronomy, Texas Christian University, 2800 South University Drive, Fort Worth, TX 76129, USA \\ ${ }^{16}$ Laboratório Interinstitucional de e-Astronomia - LIneA, Rua Gal. José Cristino 77, Rio de Janeiro, RJ - 20921-400, Brazil \\ ${ }^{17}$ Osservatorio Astronomico di Padova, INAF, Vicolo dell'Osservatorio 5, I-35122 Padova, Italy \\ ${ }^{18}$ Institut Utinam, CNRS UMR6213, OSU THETA, Université de Franche-Comté, 41 bis avenue de 1 Observatoire, F-25000 Besançon, France \\ ${ }^{19}$ Department of Astronomy and Astrophysics, The Pennsylvania State University, University Park, PA 16802, USA \\ Received 2012 November 21; accepted 2013 January 3; published 2013 March 26
}

\begin{abstract}
Despite its importance for understanding the nature of early stellar generations and for constraining Galactic bulge formation models, at present little is known about the metal-poor stellar content of the central Milky Way. This is a consequence of the great distances involved and intervening dust obscuration, which challenge optical studies. However, the Apache Point Observatory Galactic Evolution Experiment (APOGEE), a wide-area, multifiber, highresolution spectroscopic survey within Sloan Digital Sky Survey III, is exploring the chemistry of all Galactic stellar populations at infrared wavelengths, with particular emphasis on the disk and the bulge. An automated spectral analysis of data on 2403 giant stars in 12 fields in the bulge obtained during APOGEE commissioning yielded five stars with low metallicity $([\mathrm{Fe} / \mathrm{H}] \leqslant-1.7)$, including two that are very metal-poor $[\mathrm{Fe} / \mathrm{H}] \sim-2.1$ by bulge standards. Luminosity-based distance estimates place the 5 stars within the outer bulge, where 1246 of the other analyzed stars may reside. A manual reanalysis of the spectra verifies the low metallicities, and finds these stars to be enhanced in the $\alpha$-elements $\mathrm{O}, \mathrm{Mg}$, and $\mathrm{Si}$ without significant $\alpha$-pattern differences with other local halo or metal-weak thick-disk stars of similar metallicity, or even with other more metal-rich bulge stars. While neither the kinematics nor chemistry of these stars can yet definitively determine which, if any, are truly bulge members, rather than denizens of other populations co-located with the bulge, the newly identified stars reveal that the chemistry of metal-poor stars in the central Galaxy resembles that of metal-weak thick-disk stars at similar metallicity.
\end{abstract}

Key words: stars: abundances - stars: atmospheres - Galaxy: center - Galaxy: structure

Online-only material: color figures

\section{INTRODUCTION}

The chemical compositions of the oldest stars hold fundamental clues about the early history of galaxies. Even if no true Population III stars presently exist in the Milky Way (MW), their nature can be constrained from observations of the elemental abundance patterns of the most metal-poor existing Galactic stars (e.g., Beers \& Christlieb 2005; Ekström et al. 2008). Theoretical predictions suggest that the oldest, most metal-poor stars in the MW are to be found in the bulge (e.g., White \& Springel 2000; Tumlinson 2010). However, these ancient relics are extremely difficult to identify because of the combination of very high extinction, foreground contamination, and the fact that the most metal-poor stars are but a small fraction of a large population of stars located in the inner Galaxy. As a result, our view of the early stages of Galactic formation and chemical evolution has been skewed by studies of more easily accessible Galactic halo samples at large Galactocentric distances.

To date, the origin of the Galactic bulge is still uncertain. The boxy X-shape (e.g., McWilliam \& Zoccali 2010) and high metallicity (e.g., Rich 1988) of the bulge suggests secular formation associated with the disk and bar-i.e., a pseudo-bulge (e.g., Immeli et al. 2004; Kormendy \& Kennicutt 2004). On the other hand, the metallicity gradient of the bulge (Zoccali et al. 2008; Johnson et al. 2011) and its old age ( 10 Gyr; Clarkson et al. 2008) are more consistent with a classical bulge (e.g., Rahimi et al. 2010; Bournaud et al. 2011). However, the above criteria are clearly not definitive model discriminators, given that Kunder et al. (2012) also discuss metallicity gradients in a secular scenario, the high metallicity in the classical scenario 
could be explained by early starbursts (McWilliam \& Rich 1994), and old age could be understood in a secular scenario if disk instabilities occurred at early times. Moreover, recent studies by Babusiaux et al. (2010) and Hill et al. (2011) suggest that the central MW may include the superposition of a classical bulge and a pseudo-bulge.

To discriminate between these formation scenarios, a comprehensive chemical analysis of the central MW is needed to characterize the metal-poor end of its metallicity distribution function (MDF) and assess abundance patterns of bulge populations at all metallicities. The ratio between $\alpha$-element and iron abundances $\left([\alpha / \mathrm{Fe}]^{20}\right)$ of a stellar population is sensitive to the initial mass function of its parental population, whereas the position of the "knee" of the metallicity $-[\alpha / \mathrm{Fe}]$ relation is sensitive to the early star formation rate. The spread in $[\alpha / \mathrm{Fe}]$ at given $[\mathrm{Fe} / \mathrm{H}]$ depends on whether the metal-poor bulge stars were accreted or produced in situ, and by which mechanisms (Immeli et al. 2004; Rahimi et al. 2010). Moreover, in the secular instability scenario, the bulge $[\alpha / \mathrm{Fe}]$ pattern should resemble that of the inner disk. Unfortunately, this discriminatory power of chemical abundances has barely been exploited because most spectroscopic studies have been restricted to high-metallicity bulge stars. For example, the pioneering medium-resolution optical study of 12 giants by McWilliam \& Rich (1994) that discovered the bulge to be $\alpha$-enhanced (a signature of rapid formation) was limited to $[\mathrm{Fe} / \mathrm{H}] \geqslant-1.08$. Similar results were obtained from high-resolution, near-infrared spectroscopy of 14 bulge giants with $[\mathrm{Fe} / \mathrm{H}] \sim-0.33$ by Rich \& Origlia (2005), and 7 more with $[\mathrm{Fe} / \mathrm{H}] \geqslant-1.05$ by Cunha \& Smith (2006). Subsequent high-resolution optical analyses (e.g., dozens of stars by Fulbright et al. 2007; Zoccali et al. 2006; Lecureur et al. 2007) -still probing only $[\mathrm{Fe} / \mathrm{H}] \geqslant-1.5$-revealed a bulge that is more $\alpha$-enhanced than the local thick disk, although Johnson et al. (2011) find a difference that is of the order of their uncertainties and possibly due to different abundance scales. Starbursts were invoked to explain these higher $\alpha$-element levels within a classical formation scenario. However, more recent studies comparing bulge with inner disk (Bensby et al. 2010), or thick-disk stars (and with more homogeneous analyses; Meléndez et al. 2008; Ryde et al. 2010; Alves-Brito et al. 2010; Gonzalez et al. 2011) did find common abundance patterns, which supports bulge formation scenarios invoking either secular evolution or radial stellar migrations associated with spiral arms and/or the bar (Schönrich \& Binney 2009; but cf. Minchev et al. 2012).

A striking aspect of all previous spectroscopic surveys of the bulge is that despite sample sizes approaching a thousand stars, until only very recently the most metal-poor star identified had $[\mathrm{Fe} / \mathrm{H}]=-1.69$ (Zoccali et al. 2008), with only four stars having $[\mathrm{Fe} / \mathrm{H}]<-1.5$ known. Clearly, any hope of probing the extremely minor, but exceedingly interesting, metal-poor content of the central Galaxy requires much larger samples-a challenging prospect, given the significant distance and foreground dust obscuration. The situation is changing rapidly, the large ARGOS survey at medium-resolution recently reported $[\mathrm{Fe} / \mathrm{H}]$ and averaged $[\alpha / \mathrm{Fe}]$ for stars in the inner Galaxy down to $[\mathrm{Fe} / \mathrm{H}]=-2.60$ (Ness et al. 2013). Here we report the discovery of 5 additional stars with $[\mathrm{Fe} / \mathrm{H} \lesssim-1.5$ in the central Galaxy found within a sample of $\sim 2403$ stars observed in bulge fields by the Apache Point Observatory Galactic Evolution Experiment (APOGEE; Majewski et al. 2010), part of the

${ }^{20}[\mathrm{X} / \mathrm{Fe}]=A(X)-A(X)_{\odot}-\left(A(\mathrm{Fe})-A(\mathrm{Fe})_{\odot}\right), A(X)=\log \left(N_{X} / N_{\mathrm{H}}\right)+12$, where $N_{X}$ represents the number density of nuclei of element $X$.
Sloan Digital Sky Survey III (SDSS-III; Eisenstein et al. 2011), commissioning. APOGEE uses a high-resolution, $H$-band spectrograph with 300 optical fibers mated to the large field-ofview, Sloan $2.5 \mathrm{~m}$ telescope (Gunn et al. 2006). We also present detailed abundance ratios for these stars and find that they are similar to metal-poor stars in other parts of the Galaxy.

\section{OBSERVATIONS AND ABUNDANCE ANALYSIS}

APOGEE commissioning observations were taken in 2011 May-July for $\sim 4700 \mathrm{~K} / \mathrm{M}$ giant stars in 18 fields spanning $-1^{\circ}<l<20^{\circ},|b|<20^{\circ}$, and $\delta>-32^{\circ}$ (see Nidever et al. 2012, Figure 1). Stars were selected from the 2MASS Point Source Catalog (Skrutskie et al. 2006) by color $\left(\left(J-K_{\mathrm{s}}\right)_{0} \geqslant 0.5\right)$ and magnitude $(H \leqslant 11.0)$ (see G. Zasowski et al. 2013, in preparation). The observed spectra were of high quality $\left(R=22,500\right.$, signal-to-noise ratio $(\mathrm{S} / \mathrm{N})>150 \mathrm{pixel}^{-1}$, at near Nyquist sampling), although misplacement of the red detector led to degraded resolution $(R \sim 14,500)$ for $1.65 \mu \mathrm{m}<$ $\lambda<1.70 \mu \mathrm{m}$. The raw datacubes were reduced to calibrated, one-dimensional (1D) spectra and stellar radial velocities (RVs) were derived using the APOGEE reduction pipeline (Nidever et al. 2012). Effective temperatures ( $\left.T_{\text {eff }}\right)$, surface gravities $(\log g)$, and $[\mathrm{Fe} / \mathrm{H}]$ from an early version of the APOGEE Stellar Parameter and Chemical Abundances Pipeline (ASPCAP; A. E. García Pérez et al. 2013, in preparation) were used to select candidate metal-poor stars in 12 bulge commissioning fields within 10.5 from the Galactic center. Six stars were selected as having $[\mathrm{Fe} / \mathrm{H}]_{\mathrm{ASPCAP}} \leqslant-1.7$, but one was rejected from further consideration for showing peculiar line profiles (potentially a spectroscopic binary). Specific sections of the APOGEE spectra of the five metal-poor stars (Table 1) were then re-analyzed interactively via a classical 1D-LTE spectrum synthesis approach (see Figure 1). The synthesis used MARCS model atmospheres (Asplund et al. 1997), computed for the individual stellar parameters and chemical compositions listed in Table 1. Equipped with the model atmospheres, stellar spectra were synthesized using the Uppsala code BSYN and a line list (version 201202161204) compiled specifically for APOGEE (M. Shetrone et al. 2013, in preparation). Both the instrumental and macroturbulence profiles were described by Gaussians whose widths were adjusted to the variable instrumental resolution $(\lambda / \Delta \lambda=12,000-22,000)$. Following Meléndez et al. (2008), several iterations were performed to ensure consistency between the derived chemical compositions and those of the model atmospheres employed.

Initial estimates of the atmospheric parameters were based on the observed spectra, in combination with theoretical isochrones. To determine $T_{\text {eff }}$, the ratio $\left(R_{\mathrm{OH}-\mathrm{Mg}}\right)$ of the sum of $\mathrm{OH}$ line strengths (at $1.57589 \mu \mathrm{m}$ and $1.57608 \mu \mathrm{m}$ ) to that of a nearby $\mathrm{Mg}$ I line (at $1.57533 \mu \mathrm{m}$ ) was measured. ${ }^{21} R_{\mathrm{OH}-\mathrm{Mg}}$ is quite sensitive to $T_{\text {eff }}$, due to the strong temperature dependence. We calibrated $R_{\mathrm{OH}-\mathrm{Mg}}$ versus $T_{\text {eff }}$ using data for the field red giants $\alpha$ Boo, $\mu$ Leo, $\beta$ And, and $\delta$ Oph, and giants from the globular clusters M3, M13, and M71. Though spanning a large range in metallicity, age, and mass, these particular stars define an $R_{\mathrm{OH}-\mathrm{Mg}}-T_{\text {eff }}$ relation with an intrinsic scatter of only $\sim \pm 100-120 \mathrm{~K}$.

Surface gravities were derived from isochrones (Dotter et al. 2008 ) with an assumed age of $10 \mathrm{Gyr}$ and $[\alpha / \mathrm{Fe}]=+0.6$ (consistent with our final derived values). The adopted values of $T_{\text {eff }}$ and $\log g$ for the stars are given in Table 1 , and were

\footnotetext{
21 Cited wavelengths refer to vacuum measurements.
} 

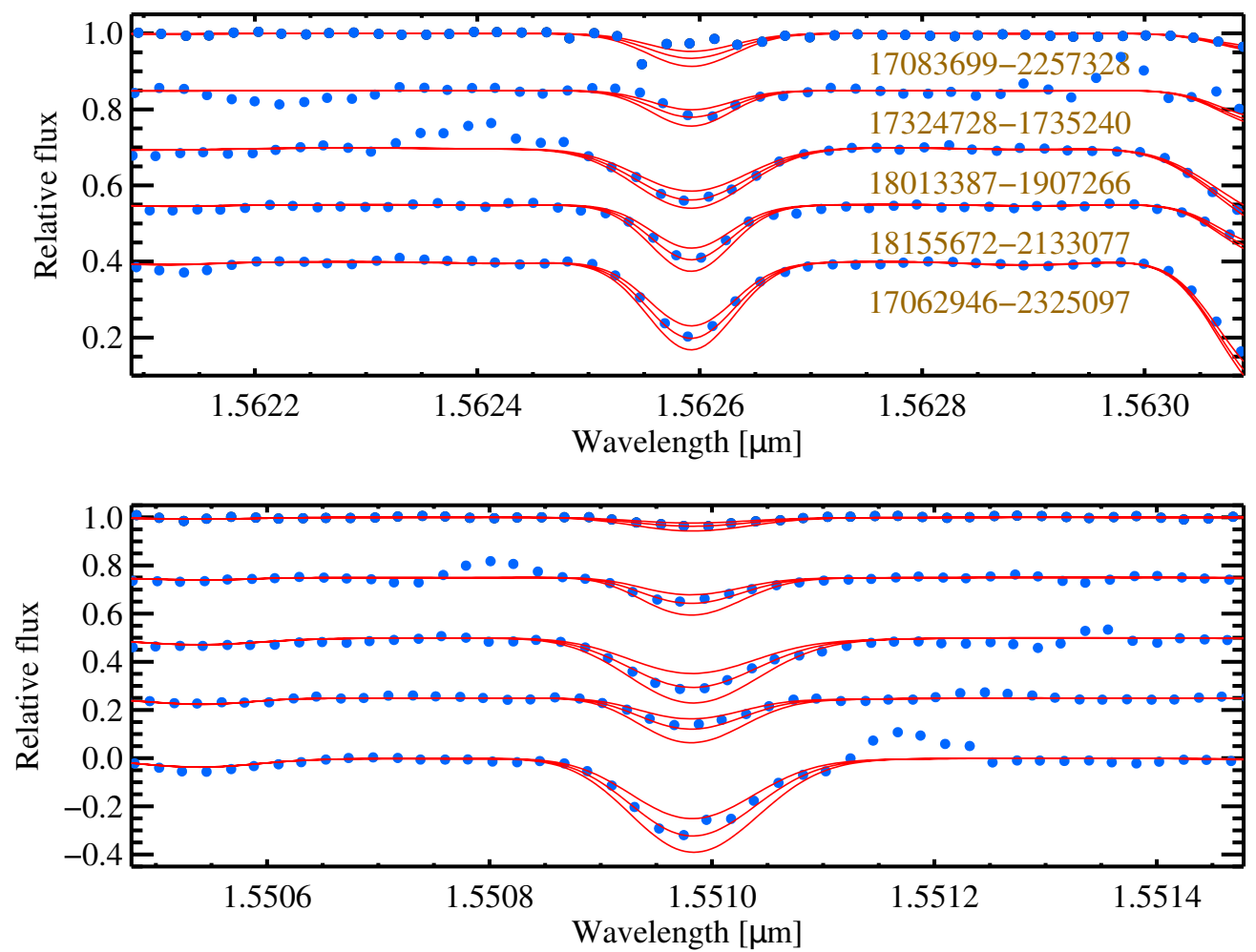

Figure 1. Observed (circles) and synthetic (solid line) spectra of the Fe I $1.56259 \mu \mathrm{m}$ (top panel) and OH lines at $\sim 1.5510 \mu \mathrm{m}$ (bottom panel) for the five metal-poor stars in our study. Spectra were offset vertically by multiples of $0.15(\mathrm{Fe})$ and $0.25(\mathrm{OH})$ for clarity. The synthesis for the best-fitting abundance and \pm 0.2 dex from that are also shown.

(A color version of this figure is available in the online journal.)

Table 1

Derived Stellar Parameters and Abundances for the Metal-poor Bulge Candidates

\begin{tabular}{|c|c|c|c|c|c|}
\hline 2MASS Star ID = & $17062946-2325097$ & $17083699-2257328$ & $17324728-1735240$ & 18013387-1907266 & $18155672-2133077$ \\
\hline$l\left(^{\circ}\right)$ & 359.727099 & 0.396365 & 8.108990 & 10.301410 & 9.811497 \\
\hline$b\left(^{\circ}\right)$ & 10.358167 & 10.228863 & 8.491406 & 1.842886 & -2.282258 \\
\hline$\alpha_{2000}(\mathrm{~h} \mathrm{~m} \mathrm{~s})$ & 170629.46 & 170836.99 & 173247.28 & 180133.87 & 181556.72 \\
\hline$\delta_{2000}(\mathrm{~h} \mathrm{~m} \mathrm{~s})$ & -232509.7 & -225732.8 & -173524.0 & -190726.6 & -213307.7 \\
\hline$H(\mathrm{mag})$ & 9.38 & 9.794 & 9.686 & 9.665 & 8.828 \\
\hline$A_{\mathrm{Ks}}(\mathrm{mag})$ & 0.319 & 0.293 & 0.247 & 0.607 & 0.275 \\
\hline$V_{\text {helio }}\left(\mathrm{km} \mathrm{s}^{-1}\right)$ & -39.49 & 328.49 & 21.03 & 142.17 & -49.79 \\
\hline$d(\mathrm{kpc})$ & 9.43 & 8.37 & 9.64 & 7.40 & 5.71 \\
\hline $\mathrm{S} / \mathrm{N}$ & 403 & 326 & 230 & 159 & 251 \\
\hline$T_{\mathrm{eff}}(\mathrm{K})$ & $3900( \pm 150)$ & $4300( \pm 150)$ & $4200( \pm 150)$ & $4000( \pm 150)$ & $4100( \pm 150)$ \\
\hline $\log g(\mathrm{cgs})$ & $0.36( \pm 0.50)$ & $0.70( \pm 0.50)$ & $0.55( \pm 0.50)$ & $0.52( \pm 0.50)$ & $0.63( \pm 0.50)$ \\
\hline$[\mathrm{Fe} / \mathrm{H}]$ & $-1.47( \pm 0.20)$ & $-2.10( \pm 0.20)$ & $-2.05( \pm 0.20)$ & $-1.54( \pm 0.20)$ & $-1.66( \pm 0.20)$ \\
\hline$\xi_{t}\left(\mathrm{~km} \mathrm{~s}^{-1}\right)$ & $3.0( \pm 0.5)$ & $2.5( \pm 0.5)$ & $2.5( \pm 0.5)$ & $2.5( \pm 0.5)$ & $2.5( \pm 0.5)$ \\
\hline$A(\mathrm{Fe})$ & $5.98( \pm 0.12)$ & $5.35( \pm 0.12)$ & $5.40( \pm 0.12)$ & $5.91( \pm 0.12)$ & $5.79( \pm 0.12)$ \\
\hline$A(\mathrm{O})$ & $7.72( \pm 0.38)$ & $7.04( \pm 0.38)$ & $7.13( \pm 0.38)$ & $7.75( \pm 0.38)$ & $7.42( \pm 0.38)$ \\
\hline$A(\mathrm{Mg})$ & $6.23( \pm 0.15)$ & $5.76( \pm 0.15)$ & $5.71( \pm 0.15)$ & $6.30( \pm 0.15)$ & $6.12( \pm 0.15)$ \\
\hline$A(\mathrm{Si})$ & $6.15( \pm 0.10)$ & $5.58( \pm 0.10)$ & $5.57( \pm 0.10)$ & $6.36( \pm 0.10)$ & $6.18( \pm 0.10)$ \\
\hline$[\mathrm{O} / \mathrm{Fe}]$ & $+0.53\left({ }_{-0.26}^{+0.28}\right)$ & $+0.48\left(\begin{array}{c}+0.28 \\
-0.26\end{array}\right)$ & $+0.52\left(\begin{array}{c}+0.28 \\
-0.26\end{array}\right)$ & $+0.63\left(\begin{array}{c}+0.28 \\
-0.26\end{array}\right)$ & $+0.42\left({ }_{-0.26}^{+0.28}\right)$ \\
\hline$[\mathrm{Mg} / \mathrm{Fe}]$ & $+0.17\left({ }_{-0.07}^{+0.09}\right)$ & $+0.33\left({ }_{-0.07}^{+0.09}\right)$ & $+0.23\left({ }_{-0.07}^{+0.09}\right)$ & $+0.31\left({ }_{-0.07}^{+0.09}\right)$ & $+0.25\left({ }_{-0.07}^{+0.09}\right)$ \\
\hline$[\mathrm{Si} / \mathrm{Fe}]$ & $+0.11\left({ }_{-0.08}^{+0.05}\right)$ & $+0.17\left({ }_{-0.08}^{+0.05}\right)$ & $+0.11\left(\begin{array}{c}+0.05 \\
-0.08\end{array}\right)$ & $+0.39\left(\begin{array}{c}+0.05 \\
-0.08\end{array}\right)$ & $+0.33\left({ }_{-0.08}^{+0.05}\right)$ \\
\hline
\end{tabular}

checked using the temperature and gravity-sensitive profiles of $\mathrm{H}$ I lines at $1.61137 \mu \mathrm{m}$ and $1.68111 \mu \mathrm{m}$, with theoretical line absorption profiles from Ali \& Griem (1966).

Stellar metallicity estimates $([\mathrm{Fe} / \mathrm{H}])$ are based on mean values of iron abundance derived from a sample of 4-13 measured Fe I lines, and assuming a solar abundance value of $A(\mathrm{Fe})_{\odot}=7.45$ (Asplund et al. 2005). For the other elements, values of $A(\mathrm{O})_{\odot}=8.66, A(\mathrm{Mg})_{\odot}=7.58$, and $A(\mathrm{Si})_{\odot}=$
7.55 (Asplund et al. 2005) were assumed. The lines were selected from the ASPCAP line list among those with minimum blending from molecular lines in the atmospheric parameter range explored in this study. Sample spectra and synthesis for the Fe I $1.56259 \mu \mathrm{m}$ line are shown in Figure 1.

Microturbulent velocities $\left(\xi_{t}\right)$ were derived by forcing consistency between the abundances obtained from weak and strong $\mathrm{Mg}$ and $\mathrm{Si}$ lines. The lines used were the following: $\mathrm{Mg}$ 


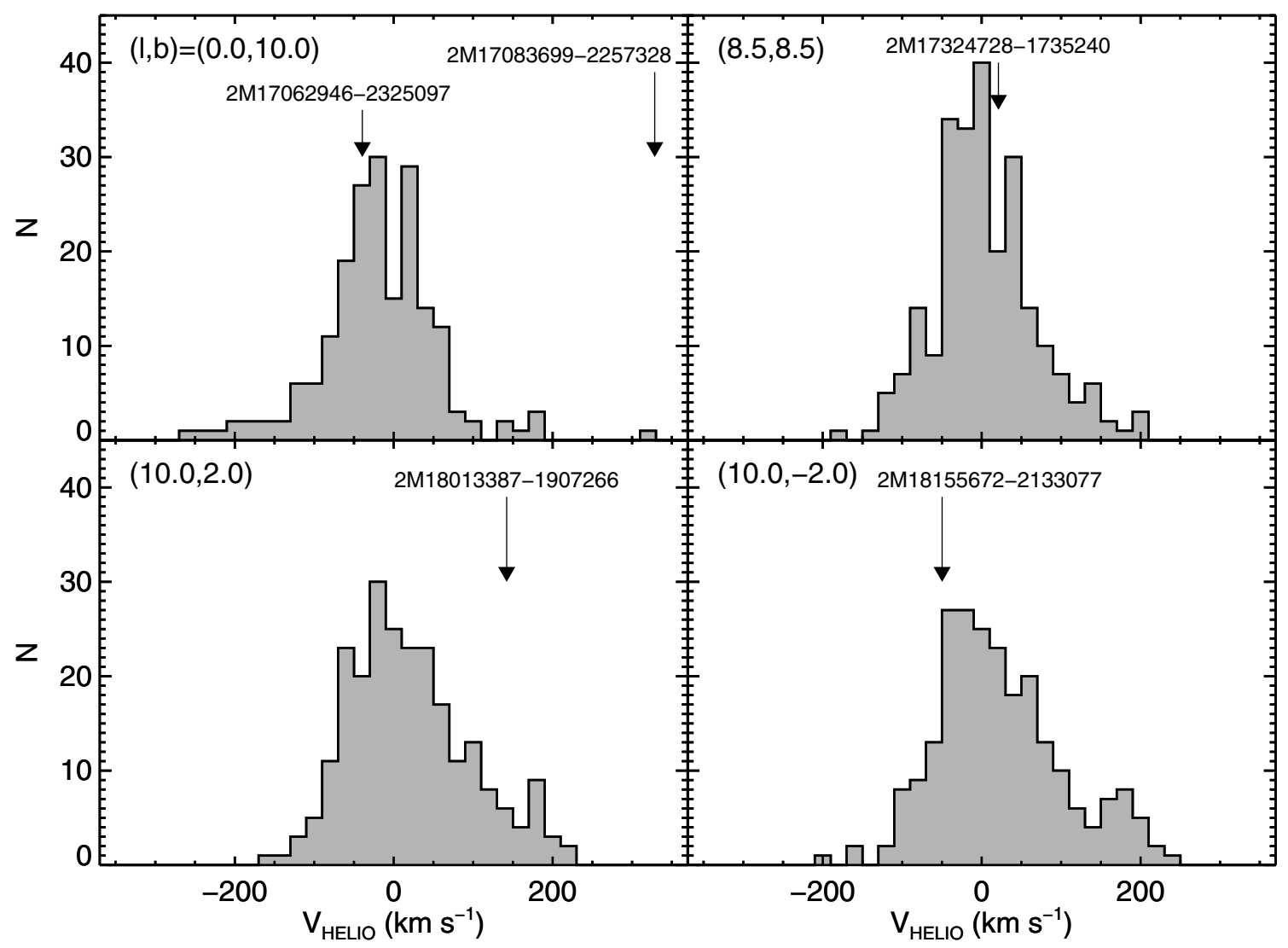

Figure 2. Heliocentric RV distributions for stars in the four observed bulge fields, with the velocities of the five metal-poor stars indicated.

Table 2

Abundance Sensitivity to Stellar Parameter Uncertainties

\begin{tabular}{lcccr}
\hline \hline & $\begin{array}{c}A \\
(\mathrm{Fe})\end{array}$ & \multicolumn{1}{c}{$\begin{array}{c}A \\
(\mathrm{O})\end{array}$} & \multicolumn{1}{c}{$\begin{array}{c}A \\
(\mathrm{Mg})\end{array}$} & \multicolumn{1}{c}{\begin{tabular}{c}
\multicolumn{1}{c}{$(\mathrm{Si})$} \\
\hline$\Delta T_{\text {eff }}(+150 \mathrm{~K})$
\end{tabular}} \\
$(-150 \mathrm{~K})$ & 0.090 & 0.276 & 0.090 & 0.072 \\
$\Delta \log g(0.5)(\mathrm{cgs})$ & -0.084 & -0.258 & -0.090 & -0.042 \\
$(-0.5)(\mathrm{cgs})$ & 0.008 & -0.110 & -0.060 & 0.000 \\
$\Delta \xi_{t}\left(0.5 \mathrm{~km} \mathrm{~s}^{-1}\right)$ & -0.008 & -0.140 & 0.050 & 0.015 \\
$\left(-0.5 \mathrm{~km} \mathrm{~s}^{-1}\right)$ & 0.016 & 0.006 & -0.080 & -0.050 \\
$\Delta[\mathrm{Fe} / \mathrm{H}](0.2 \mathrm{dex})$ & 0.003 & 0.120 & -0.080 & 0.070 \\
$(-0.2 \mathrm{dex})$ & 0.007 & -0.100 & 0.005 & 0.006 \\
\hline
\end{tabular}

$1.57450,1.57533,1.57700$, and $1.59588 \mu \mathrm{m}$, and Si I 1.59644, $1.60992,1.66853$, and $1.66853 \mu \mathrm{m}$. We obtain $\xi_{t}=2.5 \mathrm{~km} \mathrm{~s}^{-1}$ for all stars (except one with $3.0 \mathrm{~km} \mathrm{~s}^{-1}$ ).

The oxygen abundances were obtained from the analysis of $10-17 \mathrm{OH}$ lines covering $\lambda \lambda=1.5395-1.6376 \mu \mathrm{m}$. The mean abundance values are listed in Table 1 , and the observed and synthetic spectra of $\mathrm{OH}$ lines at $\sim 1.5510 \mu \mathrm{m}$ are shown in Figure 1 for all stars. Because there may be some interdependence of $\mathrm{O}$ and $\mathrm{C}$ abundances through $\mathrm{CO}$ formation, our determinations require a priori knowledge of $\mathrm{C}$ abundances, which were estimated from very weak $\mathrm{CO}$ bands, and the non-detection of the $\mathrm{C}_{\mathrm{I}}$ atomic line at $1.68950 \mu \mathrm{m}$ in any of the stars.

Internal errors in the abundances were derived from the abundance sensitivity to stellar parameters (Table 2) using the star 2M17083699-2257328 as a baseline and adopting the values listed in Table 2 as our uncertainties in the other parameters. For all elements, and oxygen in particular, abundance uncertainties are most sensitive to errors in $T_{\text {eff }}$. Overall, the abundances show moderate sensitivity to errors in $\log g$ (typically $\Delta A(X)<$ $0.1 \mathrm{dex}$ ), and are similarly or less sensitive to uncertainties in microturbulence and $[\mathrm{Fe} / \mathrm{H}]$. Final uncertainties were computed by adding the errors in quadrature and are $0.12,0.38,0.15$, and 0.10 dex for $\mathrm{Fe}, \mathrm{O}, \mathrm{Mg}$, and $\mathrm{Si}$, respectively.

\section{POPULATION MEMBERSHIP}

The stars in Table 1 have $(l, b)$ typical of the outer bulge, as do $\sim 1246$ other automatically analyzed stars in our sample, but it is unclear whether the stars in that table are actually in, and belong to, the bulge. To gauge distances, luminosities were estimated from the adopted $\log g$ and derived $T_{\text {eff }}$, assuming $M=0.8 M_{\odot}$, as expected for old giants. To determine $M_{H}$, bolometric corrections were estimated from $T_{\text {eff }}$ using a calibration derived from stellar isochrones in Girardi et al. (2000). Extinctions were estimated by combining near- and mid-IR photometry (Majewski et al. 2011) and the Indebetouw et al. (2005) extinction law. The derived distances (Table 1) have relatively large uncertainties, given the uncertainties in $T_{\text {eff }}$, gravities, assumed masses, and extinctions. Nevertheless, the distances-projected on the Galactic plane at $d / \cos (b)=5.71-9.59 \mathrm{kpc}$-place these stars marginally or completely within the nominal bulge, assuming the latter has a $\sim 2-3 \mathrm{kpc}$ radius and $\sim 8 \mathrm{kpc}$ distance. However, both the Besançon (Robin et al. 2012) and Trilegal (Vanhollebeke et al. 2009) MW models predict that APOGEE target selection in these "bulge" fields should also yield a small number of metal-poor halo stars-though the expected ratio of metal-poor halo to bulge stars is presently unconstrained because it is highly dependent on uncertain extrapolations of the halo density law to small Galactocentric radii, as well as on the unknown shape of the bulge MDF. 


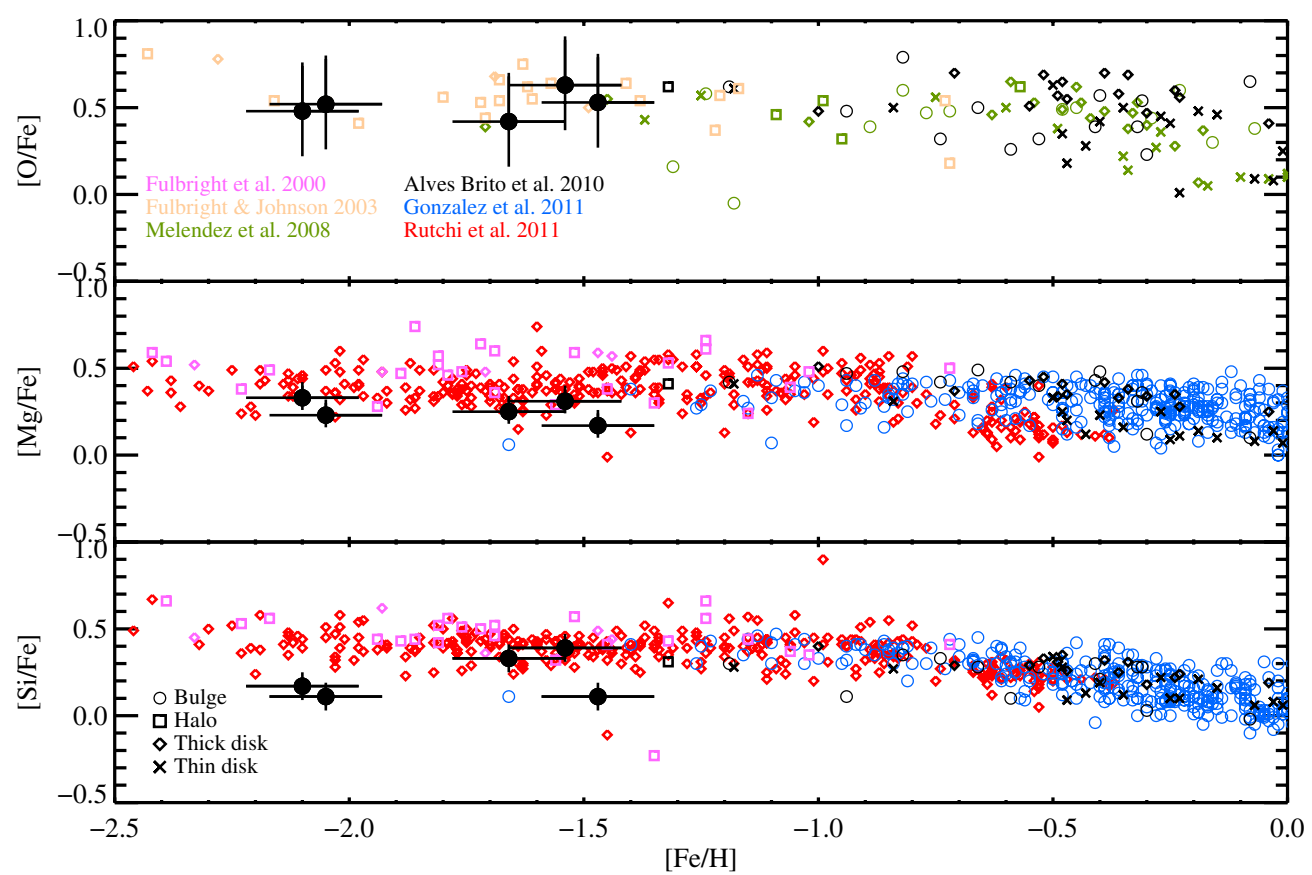

Figure 3. Comparison of our $\alpha$-element measurements of the Table 1 stars (filled circles) with those from the literature for bulge, disk, and halo stars.

(A color version of this figure is available in the online journal.)

Moreover, RVs provide little additional discrimination between bulge and overlapping halo populations because of the similar (near zero) mean velocity and comparably large velocity dispersions of the two populations. The measured RVs are generally compatible with those of more metal-rich bulge stars dominating the samples in these fields (Figure 2), although the star 2M17083699-2257328 has an extreme velocity $\left(V_{\text {hel }}=328.5 \mathrm{~km} \mathrm{~s}^{-1}\right)$ compared to bulge stars in the same field $\left(\left\langle V_{\text {hel }}\right\rangle=-18.1 \mathrm{~km} \mathrm{~s}^{-1}, \sigma_{v}=53.4 \mathrm{~km} \mathrm{~s}^{-1}\right)$ and may therefore less likely be a bulge star on dynamical grounds. We conclude that while our metal-poor stars are spatially coincident with the bulge-dominated, central Galaxy, we cannot definitively ascribe population membership to them by position or velocity.

\section{THE IRON AND $\alpha$-ELEMENT CONTENT OF THE METAL-POOR STARS}

Even if as many as four of the five stars in this study are truly bulge members, they would represent a mere $0.17 \%$ of the 2403 candidate red giant stars targeted in the 12 bulge fields. Careful detailed analysis of the data confirmed their low metallicities, with three at $[\mathrm{Fe} / \mathrm{H}] \sim-1.6$, and two at $[\mathrm{Fe} / \mathrm{H}] \sim-2.1$. The metallicities derived here are robust $([\mathrm{Fe} / \mathrm{H}]$ errors $\pm 0.11 \mathrm{dex})$, and comparable to, or lower than, the median metallicities of either local halo or metal-weak thick-disk stars, but certainly much more metal-poor than the typical bulge star. Whether they are bulge, halo, or even thick-disk members, these stars are among the most metal-poor ever found in the central parts of the Galaxy.

Our abundance estimates suggest that all five stars are $\alpha$-enhanced, with mean abundance ratios and standard deviations $[\mathrm{O} / \mathrm{Fe}]=+0.52 \pm 0.08,[\mathrm{Mg} / \mathrm{Fe}]=+0.26 \pm 0.06$, and $[\mathrm{Si} / \mathrm{Fe}]=+0.22 \pm 0.13$. Figure 3 contains these derived $[\mathrm{X} / \mathrm{Fe}]$ (along with literature values-rescaled to our assumed solar abundances-for bulge, disk, and halo stars), and shows $\mathrm{Si}$ to have the most scatter (with perhaps a hint of two [Si/Fe] subgroups), but oxygen to be most enhanced. A range of solar oxygen values exists in the literature (from different indicators and/or modeling); a different choice would have led to smaller or even larger enhancements. The $A(\mathrm{O})$ are also the most uncertain because of the great sensitivity of molecular line formation to atmospheric structure and, therefore, to the modeling employed in the spectral synthesis and to the adopted stellar parameters (especially $T_{\text {eff }}$ ). For abundance ratios, part of the sensitivity to stellar parameters is canceled out, so that the $[\mathrm{O} / \mathrm{Fe}],[\mathrm{Mg} / \mathrm{Fe}]$, and $[\mathrm{Si} / \mathrm{Fe}]$ internal errors shown in Figure 3 are reduced to ${ }_{-0.26}^{+0.28},{ }_{-0.07}^{+0.09}$, and ${ }_{-0.08}^{+0.05}$ dex, respectively.

With the exception of silicon, the $\alpha$-enhancement abundances of our sample stars are not much different from those of other, previously reported "metal-poor bulge stars" shown in Figure 3, although the latter are almost entirely at higher metallicity and exhibit significant scatter. Three of our stars exhibit lower $\mathrm{Si}$ enhancements, but comparable to those seen in the only available literature datapoint for $[\mathrm{Fe} / \mathrm{H}]<-1.5$ (Gonzalez et al. 2011). However, the latter star also apparently has a peculiar, smaller $\mathrm{Mg}$ enhancement, a feature shared with none of our stars. Data for more metal-poor stars in the Galactic bulge are needed to confirm whether these particular enhancement variations are a distinctive feature of low-metallicity stars in the bulge.

Assuming our sample stars are true bulge members, it is interesting to compare their abundances with those of thickdisk stars. Fulbright et al. (2007) and Zoccali et al. (2006), deriving large $\alpha$-enhancements for the bulge compared to published thick-disk abundances, argue for a higher star formation rate for the bulge, whereas the studies by Meléndez et al. (2008), Alves-Brito et al. (2010), and Gonzalez et al. (2011; which analyzed stars from both populations homogeneously) claim no significant $\alpha$-enhancement differences (Section 1). We can now extend these comparisons to lower metallicities using the disk data from Fulbright (2000), Fulbright \& Johnson (2003), ${ }^{22}$ and Ruchti et al. (2011). Bear in mind that such a

\footnotetext{
22 Oxygen abundances based only on the forbidden [O I] line at $630 \mathrm{~nm}$ were
} used from this source. 
comparison, especially for oxygen (see García Pérez et al. 2006), should be viewed with caution because of potential systematic errors in the analyses: different abundance and stellar parameter scales, different stellar evolutionary stages, different abundance indicators, and different locations in the Galaxy. With these caveats, we find the abundances of our stars to be comparable to those of the metal-weak thick-disk stars in Figure 3. While (1) some of our $\mathrm{Si}$ abundances may be marginally too low, and (2) there are not many metal-weak thick-disk points for comparison to our oxygen results, the metallicities and $\alpha$-element abundance patterns of our low-metallicity stars are comparable to what is found in the metal-weak thick disk. This makes a somewhat stronger case for a possible connection between the bulge and thick disk, as suggested previously, and lends further support to the notion that migrating stars populated both the Galactic bulge and thick disk (Schönrich \& Binney 2009).

The results in Table 1 indicate that these stars, as a group, do not have unusual $[\mathrm{O} / \mathrm{Fe}]$ values compared to those of halo stars, but may contain some members that have lower $[\mathrm{Si} / \mathrm{Fe}]$ and slightly lower $[\mathrm{Mg} / \mathrm{Fe}]$ values. Indeed, three of the stars have low values of $[\mathrm{Si} / \mathrm{Fe}]$ compared to most halo stars of similar metallicity, with one being 2M17083699-2257328, which has the most extreme RV and thus might be expected to be the most likely halo member. The two remaining stars cannot be chemically distinguished from local halo stars. It should be noted that Nissen \& Schuster (2010) identified a population of $\alpha$-poor halo stars. More data on the metal-poor populations of the inner Galaxy may help to disentangle possible metal-poor bulge stars from halo stars.

\section{CONCLUSIONS}

We have found five giant stars within the commissioning data of the SDSS-III's APOGEE project that have sky positions and Galactic plane-projected distances $(d / \cos (b)=5.71-9.59 \mathrm{kpc})$ expected for the bulge, but that exhibit distinctly low iron content $(-2.10 \leqslant[\mathrm{Fe} / \mathrm{H}] \leqslant-1.47)$. We present abundance ratios for these stars, significantly augmenting the sample of metal-poor bulge stars with detailed chemical information and including two stars much more metal-poor $([\mathrm{Fe} / \mathrm{H}] \sim-2.1)$ than the previous bulge star with this information (a micro-lensed dwarf with $[\mathrm{Fe} / \mathrm{H}]=-1.89$; Bensby et al. 2013), which was excluded from our comparison of only giants.

There is no strong evidence that our stars are significantly chemically different from other more metal-rich bulge stars-or even different from other stars in the halo or metal-poor thick disk, although some stars in our sample do exhibit somewhat lower Si enhancements than typically seen in other Galactic stars at these metallicities. Unfortunately, the presently available kinematics and chemistry are not sufficient to determine with certainty how many of the stars may be true bulge members. Nevertheless, this initial APOGEE sample significantly contributes to the task of compiling a more thorough census of the metal-poor stellar content of the central MW, and portends the promising results to be expected from the ongoing APOGEE exploration of the Galactic bulge.

We acknowledge funding from NSF grants AST11-09718 and AST-907873. Funding for SDSS-III has been provided by the Alfred P. Sloan Foundation, the Participating Institutions, the National Science Foundation, and the U.S. Department of Energy Office of Science. The SDSS-III Web site is http://www.sdss3.org/. SDSS-III is managed by the Astrophysical Research Consortium for the Participating Institutions of the SDSS-III Collaboration including the University of Arizona, the Brazilian Participation Group, Brookhaven National Laboratory, University of Cambridge, Carnegie Mellon University, University of Florida, the French Participation Group, the German Participation Group, Harvard University, the Instituto de Astrofisica de Canarias, the Michigan State/Notre Dame/JINA Participation Group, Johns Hopkins University, Lawrence Berkeley National Laboratory, Max Planck Institute for Astrophysics, Max Planck Institute for Extraterrestrial Physics, New Mexico State University, New York University, Ohio State University, Pennsylvania State University, University of Portsmouth, Princeton University, the Spanish Participation Group, University of Tokyo, University of Utah, Vanderbilt University, University of Virginia, University of Washington, and Yale University.

\section{REFERENCES}

Ali, A. W., \& Griem, H. R. 1966, PhRv, 144, 366

Alves-Brito, A., Meléndez, J., Asplund, M., Ramírez, I., \& Yong, D. 2010, A\&A, 513, A35

Asplund, M., Grevesse, N., \& Sauval, A. J. 2005, in ASP Conf. Ser. 336, Cosmic Abundances as Records of Stellar Evolution and Nucleosynthesis, ed. T. G. Barnes, III \& F. N. Bash (San Francisco, CA: ASP), 25

Asplund, M., Gustafsson, B., Kiselman, D., \& Eriksson, K. 1997, A\&A, 318,521

Babusiaux, C., Gómez, A., Hill, V., et al. 2010, A\&A, 519, A77

Beers, T. C., \& Christlieb, N. 2005, ARA\&A, 43, 531

Bensby, T., Alves-Brito, A., Oey, M. S., Yong, D., \& Meléndez, J. 2010, A\&A, 516, L13

Bensby, T., Yee, J. C., Feltzing, S., et al. 2013, A\&A, 549, A147

Bournaud, F., Dekel, A., Teyssier, R., et al. 2011, ApJL, 741, L33

Clarkson, W., Sahu, K., Anderson, J., et al. 2008, ApJ, 684, 1110

Cunha, K., \& Smith, V. V. 2006, ApJ, 651, 491

Dotter, A., Chaboyer, B., Jevremović, D., et al. 2008, ApJS, 178, 89

Eisenstein, D. J., Weinberg, D. H., Agol, E., et al. 2011, AJ, 142, 72

Ekström, S., Meynet, G., Chiappini, C., Hirschi, R., \& Maeder, A. 2008, A\&A, 489, 685

Fulbright, J. P. 2000, AJ, 120, 1841

Fulbright, J. P., \& Johnson, J. A. 2003, ApJ, 595, 1154

Fulbright, J. P., McWilliam, A., \& Rich, R. M. 2007, ApJ, 661, 1152

García Pérez, A. E., Asplund, M., Primas, F., Nissen, P. E., \& Gustafsson, B. 2006, A\&A, 451, 621

Girardi, L., Bressan, A., Bertelli, G., \& Chiosi, C. 2000, A\&AS, 141, 371

Gonzalez, O. A., Rejkuba, M., Zoccali, M., et al. 2011, A\&A, 530, A54

Gunn, J. E., Siegmund, W. A., Mannery, E. J., et al. 2006, AJ, 131, 2332

Hill, V., Lecureur, A., Gómez, A., et al. 2011, A\&A, 534, A80

Immeli, A., Samland, M., Gerhard, O., \& Westera, P. 2004, A\&A, 413, 547

Indebetouw, R., Mathis, J. S., Babler, B. L., et al. 2005, ApJ, 619, 931

Johnson, C. I., Rich, R. M., Fulbright, J. P., Valenti, E., \& McWilliam, A. 2011, ApJ, 732, 108

Kormendy, J., \& Kennicutt, R. C., Jr. 2004, ARA\&A, 42, 603

Kunder, A., Koch, A., Rich, R. M., et al. 2012, AJ, 143, 57

Lecureur, A., Hill, V., Zoccali, M., et al. 2007, A\&A, 465, 799

Majewski, S. R., Wilson, J. C., Hearty, F., Schiavon, R. P., \& Skrutskie, M. F. 2010, in IAU Symp. 265, Chemical Abundances in the Universe: Connecting First Stars to Planets, ed. K. Cunha, M. Spite, \& B. Barbuy (Cambridge: Cambridge Univ. Press), 480

Majewski, S. R., Zasowski, G., \& Nidever, D. L. 2011, ApJ, 739, 25

McWilliam, A., \& Rich, R. M. 1994, ApJS, 91, 749

McWilliam, A., \& Zoccali, M. 2010, ApJ, 724, 1491

Meléndez, J., Asplund, M., Alves-Brito, A., et al. 2008, A\&A, 484, L21

Minchev, I., Famaey, B., Quillen, A. C., et al. 2012, A\&A, 548, A127

Ness, M., Freeman, K., Athanassoula, E., et al. 2013, MNRAS, 430, 836

Nidever, D. L., Zasowski, G., Majewski, S. R., et al. 2012, ApJL, 755, L25

Nissen, P. E., \& Schuster, W. J. 2010, A\&A, 511, L10

Rahimi, A., Kawata, D., Brook, C. B., \& Gibson, B. K. 2010, MNRAS, 401, 1826

Rich, R. M. 1988, AJ, 95, 828

Rich, R. M., \& Origlia, L. 2005, ApJ, 634, 1293 
Robin, A. C., Marshall, D. J., Schultheis, M., \& Reylé, C. 2012, A\&A, 538, A106

Ruchti, G. R., Fulbright, J. P., Wyse, R. F. G., et al. 2011, ApJ, 737, 9

Ryde, N., Gustafsson, B., Edvardsson, B., et al. 2010, A\&A, 509, A20

Schönrich, R., \& Binney, J. 2009, MNRAS, 396, 203

Skrutskie, M. F., Cutri, R. M., Stiening, R., et al. 2006, AJ, 131, 1163
Tumlinson, J. 2010, ApJ, 708, 1398

Vanhollebeke, E., Groenewegen, M. A. T., \& Girardi, L. 2009, A\&A, 498, 95

White, S. D. M., \& Springel, V. 2000, in The First Stars, ed. A. Weiss, T. G Abel, \& V. Hill (Berlin: Springer), 327

Zoccali, M., Hill, V., Lecureur, A., et al. 2008, A\&A, 486, 177

Zoccali, M., Lecureur, A., Barbuy, B., et al. 2006, A\&A, 457, L1 